\author{
Magdalena Nigoević \\ Vana De Pol** \\ Università degli Studi di Spalato
}

\title{
POLITICAMENTE CORRETTO NEI DUE MAGGIORI QUOTIDIANI ITALIANI
}

\begin{abstract}
Questo studio si occupa del fenomeno del politicamente corretto nella lingua italiana contemporanea. Si osservano gli effetti del politicamente corretto nella lingua usata nei quotidiani italiani con i cambiamenti e le denominazioni di termini ed espressioni. A tale proposito, sono state introdotte quattro categorie principali con le loro definizioni e origini. La parte di ricerca comprende la selezione degli esempi che proverebbero o meno l'uso del politicamente corretto nella lingua italiana contemporanea. Sono stati esaminati gli articoli pubblicati nel luglio 2018 nei due quotidiani italiani online, Corriere della Sera e La Repubblica. Sono stati raccolti i termini e le espressioni: a) dell'eufemizzazione, con due sottocategorie - le disabilità delle persone e i nomi di certe professioni, b) dell'uso sessista, c) dell'uso omofobo e d) dell'uso razzista della lingua italiana. Si analizzano gli esempi estratti e si discute di quanto e in che modo certe espressioni hanno subito cambiamenti.
\end{abstract}

Parole chiave: politicamente corretto, quotidiani italiani, eufemizzazione, sessismo, omofobia, razzismo.

\section{INTRODUZIONE}

Questo studio si occupa del fenomeno del politicamente corretto nella lingua italiana. Si cercherà di dimostrare come e in quali settori si evidenzia il fenomeno del politicamente corretto e come si rispecchia la sua diffusione nella lingua italiana contemporanea. In base a ciò i diversi ambiti 'coinvolti' dal politicamente corretto in Italia verranno introdotti e spiegati con le provenienze delle espressioni e i significati dei fenomeni che riguardano le discriminazioni tra persone. I punti principali che esamineremo saranno proprio quei campi dove il politicamente corretto è rilevabile. A tale proposito si prenderà in considerazione il fenomeno dell'eufemismo perché in alcuni campi esso costituisce la parte prevalente del linguaggio politicamente

\footnotetext{
*magda@ffst.hr

**v.depol@gmail.com
} 
corretto. Seguono poi altri campi dove la correttezza politica viene applicata o meno, il sessismo, il razzismo e l'omofobia nella lingua. Per osservare il suo uso nella lingua italiana contemporanea, si trarranno gli esempi dagli articoli dei due quotidiani italiani Corriere della Sera e La Repubblica e si svolgerà l'analisi del corpus appositamente creato. In seguito, si cercherà di capire in che modo il politicamente corretto ha cambiato la lingua dei quotidiani italiani d'oggi.

\section{IL POLITICAMENTE CORRETTO}

L'enciclopedia Treccani caratterizza il termine politicamente corretto come l'espressione angloamericana politically correct (in ital. politicamente corretto - PC) che "designa un orientamento ideologico e culturale di estremo rispetto verso tutti, nel quale cioè si evita ogni potenziale offesa verso determinate categorie di persone. Secondo tale orientamento, le opinioni che si esprimono devono apparire esenti, nella forma linguistica e nella sostanza, da pregiudizi razziali, etnici, religiosi, di genere, di età, di orientamento sessuale o relativi a disabilità fisiche o psichiche della persona.". L'attenzione all'uso politicamente corretto della lingua nasce in America negli anni trenta del secolo scorso, il vero movimento si è sviluppato negli Stati Uniti e in Gran Bretagna negli anni Sessanta e in seguito si è diffuso nel resto del mondo occidentale ${ }^{2}$. Secondo Hughes (2010: 13), si tratta di un adattamento a un gruppo di opinioni liberali o radicali sulle materie sociali caratterizzato dalla difesa delle opinioni approvate e dal rifiuto del linguaggio e del comportamento considerato discriminatorio oppure offensivo. All'inizio degli anni Sessanta, negli Stati Uniti, cominciò a diffondersi l'intenzione di cancellare dalla lingua corrente usi considerati discriminatori nei confronti delle minoranze e perciò il politically correct è diventato, nella società americana, una norma linguistica più forte, in un certo senso, di quella grammaticale. All'inizio di questo fenomeno c'era l'intento di cambiare i pregiudizi sedimentati e i loro correlativi semantici introducendo nuove forme lessicali neutrali (cfr. Hughes 2010: 15-16). Con il passar del tempo e la frequente creazione di nuovi termini artificiali che dovevano sostituire quelli considerati scorretti, il politicamente corretto in molti casi ha perso la sua caratterizzazione progressista ed egualitaria, è diventata infine una versione nobilitata dell'eufemismo (cfr. Antonelli 2007: 61).

${ }^{1}$ Tratto dal sito: http://www.treccani.it/enciclopedia/politically-correct_(Enciclopedia-dell'Italiano)/ (23/06/2018).

${ }^{2}$ Sulle origini e sullo sviluppo di tale orientamento si vedano Antonelli (2007), Crisafulli (2004), Faloppa (2011) e Hughes (2010). 
Il politicamente corretto in Italia non ha assunto dimensioni eccessive e non si è diffuso capillarmente come nel mondo anglosassone, ma ha determinato un consistente cambio di mentalità e di comportamenti linguistici (cfr. Crisafulli 2004: 38). Le sue origini sono legate soprattutto all'opera di Galli de' Paratesi (1964) che si è occupata del tabù linguistico e dell'eufemismo, e di Sabatini (1987/1993) che si è occupata del sessismo della lingua italiana. Anche se non ha raggiunto un livello di obbligatorietà, ha causato modificazioni e cambiamenti nella sensibilità linguistica.

\section{METODOLOGIA}

L'analisi del linguaggio politicamente corretto nella lingua italiana sarà effettuata tenendo conto di quattro categorie principali. Anche se il fenomeno del politicamente corretto è generalmente abbastanza diffuso e concerne varie sfere del linguaggio, queste categorie sono state scelte per la loro attualità nella lingua e cultura italiana.

1) La prima categoria comprende gli esempi di eufemizzazione. Siccome si tratta di una moltitudine di 'eufemismi' che concerne varie sfere della lingua italiana, in seguito viene introdotta la suddivisione in due sottocategorie che secondo vari autori italiani che hanno discusso il PC in Italia (cfr. Galli de' Paratesi 1964, Crisafulli 2004, Canobbio 2009), hanno assunto più cambiamenti:

a) campo medico-sanitario, con degli esempi che riguardano piuttosto le disabilità, vale a dire i cambiamenti che ha assunto l'espressione 'handicappato', diventata 'portatore di handicap' e in seguito 'disabile' e che alla fine ha anche assunto delle variazioni come 'diversabile' e 'diversamente abile'. Inoltre si cercherà di verificare la frequenza delle espressioni 'sordo' - 'non udente' e 'cieco' - 'non vedente';

b) campo lavorativo con i cambiamenti dei nomi delle professioni; in particolare vengono osservati i cambiamenti nella denominazione di quattro professioni: da spazzini a netturbini a operatori ecologici, da bidelli o custodi a uscieri a collaboratori scolastici, da donna delle pulizie a collaboratrice domestica a colf, da secondini ad agenti di custodia ad agenti di polizia penitenziaria.

2) La seconda categoria si concentra sui problemi del sessismo nella lingua italiana e osserva l'accettazione delle proposte per un uso non sessista della lingua italiana. A tale scopo vengono estratti gli esempi che riguardano: a) il maschile neutro; b) l'uso di titoli professionali se il referente è femminile; c) l'uso del suffisso -essa. 
3) La terza categoria riguarda l'omofobia e la parola 'gay', nonché la questione se è più comune della parola 'omosessuale'; inoltre, si verificano anche le corrispondenti parole dispregiative, ad esempio 'frocio'.

4) Nella quarta categoria si osservano gli esempi che concernono il razzismo nella lingua italiana. L'esempio principale sarà la parola 'nero', e in base a ciò si vedrà se è più comune dell'espressione considerata politicamente più corretta 'di colore'. In questa categoria si verifica anche l'uso delle parole 'zingaro' e/o 'nomade' parlando del popolo rom.

Lo scopo dell'analisi è di osservare gli esempi delle sopraccitate categorie per poter esaminare la presenza del politicamente corretto nella lingua italiana. L'analisi sarà basata sugli esempi estratti dagli articoli giornalistici dei due quotidiani italiani online La Repubblica e Corriere della Sera nel corso di un mese - luglio del 2018. Gli articoli non appartengono a nessuna rubrica in particolare, proprio perché il fenomeno da analizzare può apparire in vari ambiti della vita quotidiana. La scelta dei giornali si giustifica con il fatto che il linguaggio politicamente corretto si è ormai diffuso ovunque, nella vita quotidiana e nei media in generale. Inoltre, $L a$ Repubblica (RP) e il Corriere della Sera (CS) sono i due quotidiani italiani più diffusi e anche, si potrebbe dire, politicamente imparziali offrendo, di conseguenza, lo specchio migliore del linguaggio quotidiano che si propone e/o, a volte, si impone.

Per il linguaggio politicamente corretto in Italia, sono stati estratti 785 esempi in totale: per la prima categoria degli eufemismi ne sono stati trovati ed estratti 212 (89 CS e $123 \mathrm{RP}$ ), per il linguaggio sessista sono stati trovati 194 esempi (106 CS e $88 \mathrm{RP}$ ); la terza categoria - l'omofobia viene rappresentata con 204 esempi (113 CS e $91 \mathrm{RP}$ ); infine, la categoria del razzismo linguistico viene esemplificata con 175 esempi (67 CS e $108 \mathrm{RP}$ ). La differenza tra il numero degli esempi estratti dai due giornali esaminati dipende per lo più dalla scelta degli argomenti da trattare nelle rispettive testate in quel periodo. Tutte le categorie hanno un numero pressappoco uguale di articoli esaminati in entrambi i quotidiani a prescindere dal loro orientamento.

\section{PRESENTAZIONE DEI DATI}

\subsection{Eufemizzazione nella lingua italiana}

L'eufemismo è "quel fenomeno linguistico per cui alcune parole vengono evitate o sostituite con altre" (Galli de' Paratesi 1964: 25) per attenuare 
l'asprezza di un concetto e usare una perifrasi o un altro vocabolo sentito come meno crudo $^{3}$. Si ricorre all'uso degli eufemismi e le versioni nobilitate per occultare contenuti sgradevoli, ad es., in resoconti mediatici sulla guerra, le locuzioni come collateral damage (danno collaterale) per 'strage di civili', 'neutralizzare il nemico' per 'uccidere', 'guerra preventiva' per 'aggressione militare' (cfr. Canobbio 2007: 42; Crisafulli 2004: 50). L'eufemizzazione è uno strumento ideale del politicamente corretto perché con la creazione di nuovi termini artificiali che devono sostituire quelli considerati scorretti ha come prodotto un linguaggio garbato e gentile (cfr. Crisafulli 2004). Si tratta di un'ampia sfera di espressioni che sono state sostituite e in tal modo rese più adeguate e meno offensive.

Nella lingua italiana l'eufemizzazione risulta più frequente nei campi che saranno presentati nelle due sottocategorie: a) le disabilità e b) il campo del lavoro vale a dire, i nomi delle professioni. Per l'analisi di questa categoria si sono estratti 212 esempi dal quotidiano Corriere della Sera e dal quotidiano La Repubblica: 128 per la prima sottocategoria e 84 per la seconda sottocategoria.

a) La prima sottocategoria si occupa di espressioni che riguardano le disabilità. Nei confronti di alcune situazioni di disagio o sofferenza si preferisce usare termini che non implicano disprezzo. Si estraggono tutti gli esempi che concernono le disabilità, si osserva la loro frequenza per vedere come sono recepite le proposte politicamente corrette. Una sensibilizzazione progressiva al problema ha portato ad una evoluzione rapidissima dell'anglicismo 'disabile' che ha sostituito la parola 'handicappato' e che è recentemente diventato 'diversamente abile' o 'diversabile' (cfr. Aprile 2005: 69-70). Inoltre, si osservano le espressioni che riguardano le disabilità uditive come 'sordo' o quella politicamente più corretta 'non udente' e le disabilità visive come' cieco' o più politicamente corretto 'non vedente'. Il numero di esempi estratto dai due quotidiani per questa sottocategoria è 128: rispettivamente, $71 \mathrm{CS}$ e $57 \mathrm{RP}$ (si veda la Tabella 1). In seguito, la percentuale dell'uso di ogni espressione estratta da entrambi i quotidiani è presentata graficamente (si vedano i Grafici 1-3).

${ }^{3}$ Secondo Galli de’ Paratesi (1964: 16) di “[...] una parola non si può dire che essa è un eufemismo in sé, ma soltanto che essa può avere un uso eufemistico. Un termine isolato dal contesto difficilmente può essere un eufemismo poiché l'essere eufemismo non è una proprietà insita nella parola, come la qualità fonetica o il genere, ma è un valore che la parola assume nell'uso che se ne fa rispetto al contesto verbale in cui la si utilizza e alla situazione in cui la si usa". 


\begin{tabular}{|c|c|c|}
\hline \multirow{2}{*}{ Espressioni per le disabilità } & Corriere della Sera & La Repubblica \\
\hline & $f$ & $f$ \\
\hline handicappato & 1 & 1 \\
\hline portatore di handicap & 3 & 5 \\
\hline disabile & 46 & 25 \\
\hline diversamente abile & l & 7 \\
\hline diversabile & / & 1 \\
\hline persone con disabilità & 11 & / \\
\hline persone con handicap & 1 & l \\
\hline non udente & 3 & 1 \\
\hline sordo & 3 & 3 \\
\hline non vedente & 1 & 5 \\
\hline cieco & 2 & 9 \\
\hline totale & 71 & 57 \\
\hline
\end{tabular}

Tabella 1. Espressioni per le disabilità nei due quotidiani esaminati

Dagli esempi che riguardano le disabilità (si veda il Grafico 1) si può concludere che in entrambi i quotidiani è preferibile l'uso della parola 'disabile' della quale è stato raccolto il maggior numero di occorrenze rispetto agli altri esempi. Questa espressione è anche il prodotto del politicamente corretto, ma ovviamente ben recepita nella lingua italiana e frequentemente usata (es. 1). Sono stati riscontrati solo due esempi dell'espressione 'handicappato' (es. 2), considerato politicamente scorretto, che viene presentato nei quotidiani solo nelle citazioni di persone che parlano delle proprie disa- 
bilità. Siccome l'espressione 'handicappato' è ormai diventata spregiativa, 'portatore di handicap' si rileva come espressione per una persona che non ha un handicap come parte costitutiva, ma come qualcosa che "porta" in più con sé4. L'espressione 'diversamente abile' con la connotazione - dotato di differenti capacità, è ritenuta esagerata perché coniata sotto influsso delle proposte del mondo anglofono ${ }^{5}$. 'Diversabile' è un neologismo composto dalla locuzione diversamente abile ${ }^{6}$. Entrambi, come si vede nella Tabella 1 , sono poco frequenti nei quotidiani presi in considerazione. Invece l'espressione 'portatore/-trice di handicap' (es. 3) è più presente. Leggendo i quotidiani si è notato che si usa spesso anche l'espressione 'persone con disabilità' (es. 4) e un po' meno l'espressione 'persone con handicap'. Questo probabilmente si deve al fatto che nella lingua inglese la preposizione with è l'unica preposizione disponibile per una persona con una malattia e perciò anche nella lingua italiana si crea il calco con la preposizione 'con'.

(1) Manca la pedana e la madre disabile ${ }^{7}$ non riesce a salire sul bus [...] (CS 1/7/2018)

(2) [...] spiega Elisa Gallo, che nel gruppo cura la comunicazione, "trasportare ad esempio un handicappato alle feste di quartiere [...]" (RP 1/8/2018)

(3) $[. .$.$] ha raccontato di non aver notato che la ragazza fosse portatrice$ di handicap. (RP 24/7/2018)

(4) [...] una mappa delle spiagge accessibili alle persone con disabilità [...] $(\mathrm{CS} \mathrm{19/7/2018)}$

${ }^{4}$ A partire dagli anni Ottanta in Italia è stato considerato inopportuno usare la parola handicappato. Si preferivano i termini persona handicappata o persona portatore di handicap "proprio per evidenziare che l'handicap era qualcosa in più, come fardello [...] che non faceva parte della persona." (Tratto dal sito: $<$ http://www.disabilitaintellettive. it/index.php?option=com_content\&task=view\&id=398\&Itemid=165>, 7/2/2019).

${ }^{5}$ Viene anche criticata spesso proprio dalle persone disabili che preferiscono l'espressione persona con disabilità così come è stata dichiarata dalla Convenzione dell'Organizzazione delle Nazioni Unite. Si veda: < http://www.cpaonline.it/carica/pubblicazioni/ConvenzioneONUdefinitivo.pdf $>$ (7/2/2019).

${ }^{6} \mathrm{Si}$ tratta di una rivisitazione della parola disabile che è stata proposta da Claudio Imprudente, Presidente dell'Associazione "Centro Documentazione Handicap" di Bologna e direttore editoriale della rivista HP Accaparlante, che la giustifica in questo modo: "A livello formale cambia solo un prefisso, ma significa spostare l'accento dalle "non abilità" alle "abilità diverse", contribuendo a cambiare la cultura del dis-valore e a passare a una logica del valore diverso." Tratto dal sito: <http://www.grusol.it/informazioni/25-0703. $\operatorname{asp}>(7 / 2 / 2019)$.

${ }^{7} \mathrm{Il}$ corsivo negli esempi è nostro. 


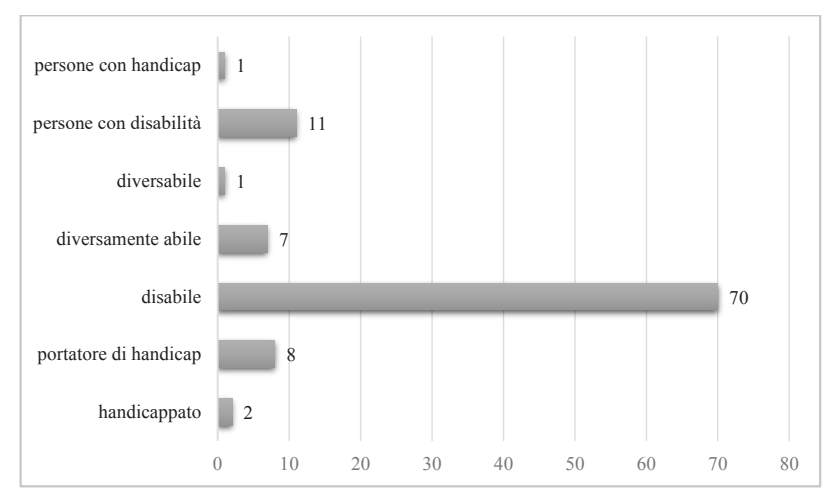

Grafico 1. Espressioni per la disabilità (in generale)

Per quanto riguarda le espressioni 'non udente' (es. 5) al posto di 'sordo' (es. 6) e 'non vedente' al posto di 'cieco', è ovvio che con la anteposizione della negazione 'non' si ottiene l'effetto di eufemizzazione. In questo modo non si stigmatizza una persona per essere 'sorda' o 'cieca' ma per essere semplicemente diversa da quella che sente e vede. Così si evita la connotazione di persona "normale" e "non normale" sorda o cieca. Per quanto riguarda i nostri dati, si può osservare che nello stile giornalistico si usano le espressioni politicamente corrette (si vedano il Grafico 2 e il Grafico 3), ma c'è una sottile preferenza per le espressioni 'sordo' $(60 \%)$ e 'cieco' (65\%) perché ovviamente non sono percepite come espressioni con forte connotazione negativa. Inoltre, alcuni studiosi italiani del politicamente corretto considerano le espressioni 'non udente' e 'non vedente' come prodotti di esagerazione (cfr. Crisafulli 2004).

(5) $\mathrm{Ci}$ avete mai pensato che un non udente ha bisogno di una sveglia che vibra [...] (CS 4/7/2018)

(6) È nato sordo Luca, ma ha avuto genitori bravi e attenti [...] (CS 4/7/2018)

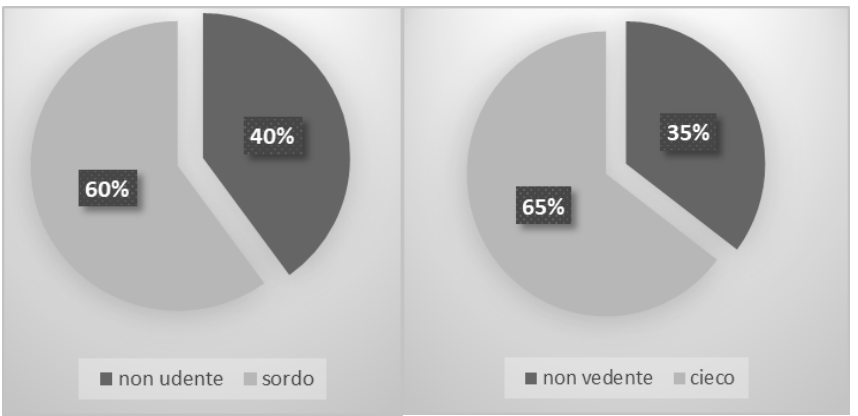


b) La seconda sottocategoria tratta le espressioni del mondo del lavoro, vale a dire i nomi delle professioni. Secondo gli autori italiani che hanno discusso il politicamente corretto, questo sarebbe forse il campo nel quale sono avvenuti più cambiamenti. La correttezza politica in Italia ha toccato particolarmente la sfera semantica legata al mondo del lavoro, in cui una serie di professioni ha cambiato nome. Nei nomi di professione sono state inventate denominazioni nuove, si nota l'influsso dell'inglese e l'abuso del linguaggio tecnico (collaboratore, operatore, addetto a qualcosa). Le trasformazioni avvengono nel caso degli 'spazzini' che diventano 'netturbini' e poi 'operatori ecologici', e in qualche caso il nome coincide con cambiamenti piccoli o grandi nella professione stessa, ma ciò appartiene ad una rivalutazione del ruolo nella società d'oggi. Molte sono le categorie di lavoratori che hanno cambiato nome, per es. i 'fornai' diventano 'panificatori', 'le badanti' 'assistenti domiciliari' o 'lavoratrici di cura', al posto del 'becchino' troviamo 'operatore funebre' o 'addetto al trasporto funebre' e così via.

La presentazione che segue si riferisce al cambiamento di quattro diversi tipi di professioni. Precisamente si tratta delle professioni prima svolte da 'spazzini' e poi 'netturbini' che sono diventati 'operatori ecologici'; da 'bidelli' a 'uscieri' a 'collaboratori scolastici'; da 'donne delle pulizie' a 'collaboratrici domestiche' a 'colf'; da 'secondini' ad 'agenti di custodia' diventati 'agenti di polizia penitenziaria'. Il numero di esempi per questa sottocategoria estratto dai due quotidiani è $84: 18$ CS e 66 RP (si veda la Tabella 2).

\begin{tabular}{|c|c|c|}
\hline \multirow{2}{*}{ Nomi delle professioni } & Corriere della Sera & La Repubblica \\
\cline { 2 - 3 } & $f$ & $f$ \\
\hline spazzino & $/$ & 9 \\
\hline netturbino & $/$ & 10 \\
\hline operatore ecologico & 2 & 9 \\
\hline \multicolumn{2}{|c|}{} \\
\hline bidello & $/$ & 6 \\
\hline usciere & $/$ & 2 \\
\hline collaboratore scolastico & $/$ & 3 \\
\hline
\end{tabular}




\begin{tabular}{|c|c|c|}
\hline donna delle pulizie & 1 & 2 \\
\hline collaboratrice domestica & 3 & 4 \\
\hline colf & 7 & 15 \\
\hline secondino & / & 1 \\
\hline agente di custodia & / & / \\
\hline agente di polizia penitenziaria & 5 & 5 \\
\hline totale & 18 & 66 \\
\hline
\end{tabular}

Tabella 2. Nomi delle professioni nei due quotidiani esaminati

Si può osservare il cambiamento in tutti e quattro i tipi di professioni. Il numero degli esempi estratto da La Repubblica è tre volte maggiore di quello estratto dal Corriere della Sera. La discrepanza nel numero tra i due quotidiani dipende dai diversi argomenti trattati nei quotidiani in quel periodo, vale a dire dipende dalla menzione delle persone che svolgono le professioni prese in considerazione. Per la stessa ragione, alcune espressioni non sono state trovate in tutti e due i quotidiani, come si vede dalla Tabella 2. Per quanto riguarda i cambiamenti dei nomi, va detto che il politicamente corretto "non migliorerà le condizioni lavorative dell'operatore ecologico, ma può influenzare la percezione, spesso negativa, che ne ha la gente: lo spazzino è uno che fa un lavoro degradante perché raccoglie l'immondizia; l'operatore ecologico è un lavoratore come un altro che si prende cura dell'ambiente in cui viviamo." (Crisafulli 2004: 42). Per tale ragione 'operatore ecologico' si utilizza molto negli annunci di lavoro, negli articoli giornalistici sostituendo l'espressione 'spazzino' o 'netturbino'. Tra l'altro il cambiamento di nome in questo caso coincide con alcuni cambiamenti nella professione stessa; questo tipo di lavoro non si svolge più nello stesso modo come si faceva in passato e perciò è anche opportuno cambiarne il nome. Dai dati ottenuti che si riferiscono a questa professione, si vede che 'operatore ecologico' è entrato nell'uso frequente ma anche lo 'spazzino' e il 'netturbino' rimangono nell'uso quotidiano (si veda la Tabella 3). 
(7) $[\ldots]$ i bidelli sono stati messi a pitturare le porte in alluminio. (RP 22/7/2018)

(8) Colf, badanti e fattorini sono i principali settori lavorativi [...] (RP 4/7/2018)

\begin{tabular}{|c|c|c|c|c|c|c|c|c|c|c|c|}
\hline \multicolumn{12}{|c|}{ Professione } \\
\hline nome & $f$ & $\%$ & nome & $f$ & $\%$ & nome & $f$ & $\%$ & nome & $f$ & $\%$ \\
\hline spazzino & 9 & 30 & bidello & 6 & 55 & $\begin{array}{c}\text { donna } \\
\text { delle } \\
\text { pulizie }\end{array}$ & 3 & 9 & $\begin{array}{l}\text { secon- } \\
\text { dino }\end{array}$ & 1 & 9 \\
\hline $\begin{array}{c}\text { netturbi- } \\
\text { no }\end{array}$ & 10 & 33 & usciere & 2 & 18 & $\begin{array}{c}\text { colla- } \\
\text { bora- } \\
\text { trice } \\
\text { dome- } \\
\text { stica }\end{array}$ & 7 & 22 & $\begin{array}{c}\text { agente } \\
\text { di } \\
\text { custo- } \\
\text { dia }\end{array}$ & 0 & 0 \\
\hline $\begin{array}{l}\text { operatore } \\
\text { ecologico }\end{array}$ & 11 & 37 & $\begin{array}{c}\text { colla- } \\
\text { bora- } \\
\text { tore } \\
\text { scola- } \\
\text { stico }\end{array}$ & 3 & 27 & colf & 22 & 69 & $\begin{array}{c}\text { agen- } \\
\text { te di } \\
\text { polizia } \\
\text { peni- } \\
\text { tenzi- } \\
\text { aria }\end{array}$ & 10 & 91 \\
\hline$\sum$ & 30 & 100 & & 11 & 100 & & 32 & 100 & & 11 & 100 \\
\hline
\end{tabular}

Tabella 3. Nomi di professione

I 'bidelli' (es. 7) sono rimasti nell'uso comune e per quanto riguarda il materiale analizzato sono più frequenti $(55 \%)$ dei 'collaboratori scolastici' $(27 \%)$ e degli 'uscieri' (18\%), caduti quasi in disuso. La 'donna delle pulizie' ha un uso minore rispetto a 'colf' (es. 8) che è l'attuale denominazione della 'collaboratrice domestica' e compare così anche nella maggioranza degli esempi estratti. I 'secondini' (9\%) da un po' di tempo sono diventati 'agenti della polizia penitenziaria' (91\%) per sottolineare il fatto che anche i 'secondini' sono poliziotti come tutti gli altri, solo con una specializzazione diversa. Questo cambiamento è stato ben recepito ed entrato nell'uso comune (si veda la Tabella 3). 


\subsection{Uso sessista della lingua italiana}

L'atteggiamento sessista si manifesta quando un genere e/o sesso ${ }^{8}$ viene considerato superiore rispetto all'altro. Oltre all'atteggiamento di superiorità, esiste anche l'avversione o l'antipatia verso il sesso opposto e persino l'attribuzione di qualità positive o negative a una persona in base al sesso. Il termine sessismo è stato coniato negli anni Sessanta del Novecento dai movimenti femministi che cercavano di stabilire l'uguaglianza tra gli uomini e le donne. È evidente che la lingua si fonda su un principio maschilista perché "nella loro qualità di gruppo dominante, gli uomini hanno costruito una realtà sociale e culturale a loro immagine e somiglianza" (Crisafulli 2004: 57). Ma, come sostiene Sabatini (1993: 21), il problema non sono le differenze tra gli uomini e le donne "ma le valenze che esse esprimono: o nozioni stereotipate, riduttive o restrittive dell'immagine della donna, o il reiterato e pervasivo concetto base della centralità e universalità dell'uomo e della marginalità e parzialità della donna".

Il problema del sessismo nella lingua è stato esaminato da vari punti di vista e soprattutto dai linguisti inglesi e statunitensi (cfr. Lepschy 1989; Lepschy, Lepschy e Sanson 2001). Per quanto riguarda l'Italia, queste idee si sono diffuse più tardi, e oggi godono dell'appoggio del governo e delle istituzioni. La prima opera più elaborata è stata pubblicata dalla linguista Alma Sabatini per la Presidenza del Consiglio nel 1987 e per la Commissione Nazionale per la parità e le pari opportunità tra uomo e donna - Le "Raccomandazioni" per un uso non sessista nella lingua italiana9. Il documento raccomanda delle alternative compatibili con il sistema della lingua per evitare alcune forme sessiste e per stabilire un vero rapporto tra valori simbolici nella lingua e valori concreti nella vita (cfr. Aprile 2005: 71). Le proposte di Sabatini si possono riassumere in 5 categorie princi-

${ }^{8}$ I termini 'sesso' e 'genere' non sono sempre ben definiti e si usano spesso come sinonimi. In questa sede non approfondiamo ulteriormente la questione, ma accenniamo soltanto che il sesso è l'insieme di tutte le caratteristiche biologiche e fisiche che contraddistinguono l'essere femmine o maschi (o persone intersessuali). Invece, per designare la specie umana, a meno che non si tratti della biologia, si dovrebbero usare i termini del genere: maschile e femminile. Il genere è una categoria culturale e psicologica, variabile secondo i contesti e le esperienze individuali (cfr. Galić 2011: 10-11).

${ }^{9}$ Il testo delle Raccomandazioni, scaricabile dal sito internet del Ministero per la Pubblica Amministrazione <http://www.funzionepubblica.gov.it/sites/funzionepubblica. gov.it/files/documenti/Normativa\%20e\%20Documentazione/Dossier\%20Pari\%20opport unit\%C3\%A0/linguaggio_non_sessista.pdf> (25/02/2019), era l'appendice di una ricerca intitolata Il sessismo nella lingua italiana, che mostrava alcuni degli aspetti di sessismo presenti in italiano. Oltre ad Alma Sabatini, vi collaborarono Marcella Mariani, Edda Billi e Alda Santangelo. 
pali: la raccomandazione di non usare il maschile come genere marcato (il maschile neutro), evitare di usare l'articolo con i cognomi femminili, accordare aggettivi e participi con i nomi che sono in maggioranza, usare $\mathrm{i}$ titoli professionali al femminile se il referente è femminile e infine evitare di usare il suffisso -essa per il suo valore ironico e discriminante ${ }^{10}$. Dalla pubblicazione delle Raccomandazioni, la discussione sulla relazione tra uso della lingua e parità di genere in Italia ha offerto diversi spunti di riflessione ${ }^{11}$. Il testo è "tutt'oggi il principale tentativo istituzionale di dare indicazioni concrete per l'utilizzo di un linguaggio rispettoso dei generi, al quale si continua a fare riferimento" (Fornara 2009: 154) ${ }^{12}$.

L'uso sessista nella lingua italiana è assai diffuso ed incorporato nella lingua al livello grammaticale e lessicale. Dalle proposte per un uso non sessista della lingua italiana di Sabatini (1987/1993), si è scelto di osservare tre sottocategorie in cui l'uso sessista si rileva quotidianamente: a) il maschile neutro; b) l'uso dei titoli professionali se il referente è femminile e c) l'uso del suffisso -essa. Anche se in un mese se ne potrebbero estrarre tantissimi esempi, per la nostra analisi abbiamo estratto un numero corrispondente alle altre categorie, in totale 194: CS 106 e RP 88.

a) Il maschile neutro

Sull'uso del maschile neutro abbiamo scelto di osservare i 44 esempi ricavati dal Corriere della Sera e i 39 esempi da La Reppublica. Questa sottocategoria, rispetto alle altre due, presenta il maggior numero di esempi perché ogni giorno si potrebbero trovare in abbondanza esempi del maschile neutro in quasi ogni articolo consultato (es. 9, 10, 11).

(9) Non c'era bambino o uomo fatto, a Linosa, che non indossasse una maglietta con la tartaruga caretta dell'Hydrosphera. (CS 24/7/2018)

(10) Il primo sottolineava la necessità che l'Islam riconoscesse la legge positiva e firmasse la dichiarazione dei diritti dell'uomo dell'Onu [...] (RP 11/7/2018)

(11) Non tanto, e non solo, la sostituzione del dipendente con la macchina ma la «robotizzazione dell'uomo-lavoratore». (RP $11 / 7 / 2018)$

${ }^{10}$ Il testo di Sabatini è abbastanza discusso, citato e criticato per cui in questa sede non ci soffermiamo ad approfondirlo. Per un esame critico delle Raccomandazioni di Alma Sabatini si veda per esempio Lepschy (1989) e Robustelli (2000, 2012).

${ }^{11}$ Per una rassegna dei contributi sul sessismo linguistico in Italia si veda Lepschy, Lepschy e Sanson (2001) e Robustelli (2000, 2018).

12 Si veda, per esempio, Robustelli (2012) - è una guida per l'uso del genere nel linguaggio amministrativo con raccomandazioni in forma rinnovata e approvata dall'Accademia della Crusca. 
Osservando gli esempi (9-11) è evidente il principio androcentrico, cioè l'uomo ha una valenza che si può riferire sia al maschio che alla femmina della specie umana, mentre la donna si riferisce soltanto alla femmina della specie. Infatti, la maggioranza degli esempi si riferisce all'uso della parola 'uomo' oppure 'uomini' con valore generico, il che non rappresenta la parità linguistica tra $\mathrm{i}$ due generi. Questo non è un dato di fatto solo della lingua italiana, ma anche di molte altre lingue. La stessa immagine del mondo androcentrico è rivelata dagli esempi dell'uso del maschile neutro quando si parla di categorie o gruppi (es. 12-14).

(12) Oltre ai bibliotecari, sono mano a mano venuti a mancare amministrativi, informatici, operatori alla vigilanza, restauratori ed assistenti tecnici. (CS 24/7/2018)

(13) «Da adesso chi tace è complice», scrive il mensile, che ha chiesto a musicisti, attori, scrittori, e altre figure del mondo dello spettacolo di prendere posizione. (CS 5/7/2018)

(14) Gli studenti di classici, scientifici ed ex magistrali siciliani riescono a competere [...] (RP 10/7/2018)

Si nota inevitabilmente l'uso comune del plurale maschile quando si parla di gruppi o categorie e lo si definisce come "maschile neutro". Ma, come scrive Robustelli (2012: 4-5), "si tratta di una definizione davvero infelice: il genere grammaticale maschile è, appunto, maschile, ed evoca esseri maschili", aggiungendo che è più opportuno parlare di "maschile inclusivo" perché viene usato, indifferentemente, per uomini e donne. Con tale abitudine linguistica, che dal linguaggio burocratico-amministrativo si estende nella lingua quotidiana, si rischia di cancellare completamente la presenza delle donne in tutti i tipi di testi. Sarebbe opportuno sostituire, ove possibile, il maschile inclusivo con le espressioni che descrivono il mestiere appropriato sia per l'uomo che per la donna, vale a dire usare anche i femminili relativi alle professioni e alle cariche che le donne assumono (per es.: ministri e ministre, attori e attrici, studenti e studentesse).

b) L'uso dei titoli professionali se il referente è femminile

Sono stati raccolti 58 esempi di questa sottocategoria: 36 CS e 22 RP. È stato estratto un numero maggiore dal quotidiano Corriere della Sera per questa sottocategoria, ma in entrambi si nota un uso frequente del maschile quando si descrive una professione femminile; in più, non è stata riscontrata nessuna differenza significativa tra i due giornali (es. 15, 16).

(15) Il Comune di Torino ha chiesto di costituirsi parte civile nel processo a Silvana De Mari, 64 anni, medico, accusata di diffamazione per aver equiparato l'omosessualità a una malattia. (CS $19 / 7 / 2018)$ 
(16) Viraj rivela l'incontro avvenuto venerdì con l'assessore Alessandra Sardu. (RP 31/7/2018)

Alcuni mestieri erano in passato riservati solo agli uomini e non esisteva un equivalente femminile. Spesso la donna non aveva il diritto di svolgerli perché questo "prestigio" apparteneva solo agli uomini. Comunque, questa situazione è cambiata e di conseguenza si dovrebbe stare attenti alla lingua che si usa. Oggi anche le donne svolgono le stesse funzioni, e in base a ciò si dovrebbero adeguare i nomi dei mestieri e si dovrebbe evitare di usare il titolo al maschile, quando si parla dei mestieri e delle professioni che svolgono le donne. Alcune professioni hanno una regolare forma al femminile e non è opportuno usarle al maschile, per esempio: il sindaco - la sindaca, lo scrittore - la scrittrice ecc.

c) L'uso del suffisso -essa

Sono stati raccolti 53 esempi di questa sottocategoria 26 estratti dal Corriere della Sera e 27 da La Repubblica: come per la sottocategoria precedente - in entrambi i quotidiani si possono trovare numerosi esempi sull'uso del suffisso - essa, senza differenze significative (es. 17-18).

(17) Era il 1968 quando Anna Galizia Danovi, giovane avvocatessa, si occupò di diritto di famiglia per la prima volta. (CS 12/7/2018)

(18) Scrittrice, poetessa e saggista Dacia Maraini è ospite a La bella estate, rassegna curata da Alba Donati, con il suo romanzo Tre donne. (RP 14/7/2018)

"Si tratta di forme femminili [NdA. il sindaco/la sindachessa, l'avvocato/l'avvocatessa] ora in disuso che hanno ormai assunto una connotazione ironica e scherzosa o addirittura spregiativa. Attualmente si preferisce perciò usare la forma maschile quando ci si riferisce a una donna" (Sensini 2009: 99). Inoltre, le alternative appropriate per ogni sostantivo che si riferisce al genere maschile esistono e si dovrebbero recepire (l'avvocatessa - l'avvocata, la poetessa - la poeta). Alcuni dei termini col suffisso -essa come 'dottoressa', 'professoressa' o 'studentessa' si sono affermati "nella prima metà del Novecento, grazie alla standardizzazione del linguaggio scolastico, e alla normalità dei riferimenti a donne laureate, insegnanti e allieve" (Lepschy, Lepschy e Sanson 2002: 406) e sono ormai entrati nell'uso quotidiano a tal punto che oggi può anche sembrare strano non usarli in un altro modo. 


\subsection{Uso omofobo della lingua italiana}

L'omofobia viene definita come un atteggiamento negativo verso le persone omosessuali ossia la "paura dell'omosessualità, nel senso di timore ossessivo di essere o scoprirsi omosessuali o di avversione patologica all'omosessualità o di entrambe le cose"13.

In seguito si osserva l'uso delle parole 'gay' e 'omosessuale' nei due quotidiani. Si nota la frequenza dei due termini prendendo in considerazione che l'espressione 'gay' è quella considerata politicamente più corretta. Per questa categoria sono stati estratti 204 esempi nel corso del mese di luglio 2018: CS 113 e RP 91. Nel quotidiano Corriere della Sera abbiamo trovato 83 esempi della parola 'gay', 25 esempi della parola 'omosessuale' e 5 esempi della parola dispregiativa 'frocio', invece nel quotidiano $\mathrm{La}$ Repubblica abbiamo trovato 53 occorrenze della parola 'gay', 34 della parola 'omosessuale' e 4 della parola 'frocio'. Già dal numero degli esempi estratti si vede che in entrambi i quotidiani la parola 'gay' (es. 19) è più frequente della parola 'omosessuale' (es. 20). Ci sono vari esempi dove il termine 'gay' appare spesso come parte di un nome o un sintagma, quali: 'Gay Pride', 'Gay Center' (es. 21), 'gay street', ecc.

(19) È davvero assurdo che nel 2018 un ragazzo, gay o etero, non sia libero di vestirsi come vuole o di camminare come vuole. (CS $25 / 7 / 2018$ )

(20) $[. .$.$] a riconoscere ai componenti delle coppie omosessuali di$ adottare il figlio del partner, così diventando anche coppia genitoriale. (RP 31/7/2018)

(21) Dopo lo scontrino con la parola «fr...» ai due omosessuali e il licenziamento in tronco del cameriere "colpevole», il Gay Center continua a chiedere la revoca [...]. (CS 22/7/2018)

Nel vocabolario Treccani online si legge che la parola ' gay' originariamente portava il significato di "allegro". La stessa parola a partire degli anni Sessanta negli Stati Uniti esprimeva "l'orgoglio di chi si è dato un'identità alternativa" (Crisafulli 2004: 69) e in seguito si è estesa ad altre lingue e ha perso quasi completamente le connotazioni specifiche, diventando oggi un'espressione neutra. Secondo alcuni autori l'espressione 'omosessuale' stigmatizza un certo comportamento e la si vede come una condizione di malattia (cfr. Crisafulli 2004). I dati ottenuti dagli articoli analizzati dei due quotidiani presi in considerazione confermano la prevalenza della parola 'gay' (67\%) rispetto alle altre espressioni (si veda il Grafico 4). Comunque,

${ }^{13}$ Tratto dal dizionario Treccani $<$ http://www.treccani.it/enciclopedia/omofobia_res3c4ed4e0-9b55-11e1-9b2f-d5ce3506d72e_(Dizionario-di-Medicina)/> (7/2/2019). 
in molti articoli vengono usate tutte e due le parole indipendentemente: per esempio si vede 'gay' nel titolo ${ }^{14}$ e poi sia 'gay' che 'omosessuale' nel contenuto dell'articolo. Del resto i termini spregiativi come 'frocio' sono molto rari (solo 4\%), si trovano solo negli esempi che citano la vita privata delle persone parlando di avvenimenti e situazioni sgradevoli. Questa parola è portatrice di un significato assai spregiativo e degradante il che si nota anche dal modo in cui viene riportata nei quotidiani, vale a dire in alcune situazioni non viene scritta l'intera parola ma si ricorre all'uso dei puntini o/e si scrive tra virgolette (es. 21).

\section{Grafico 4. Espressioni per l'uso omofobo}

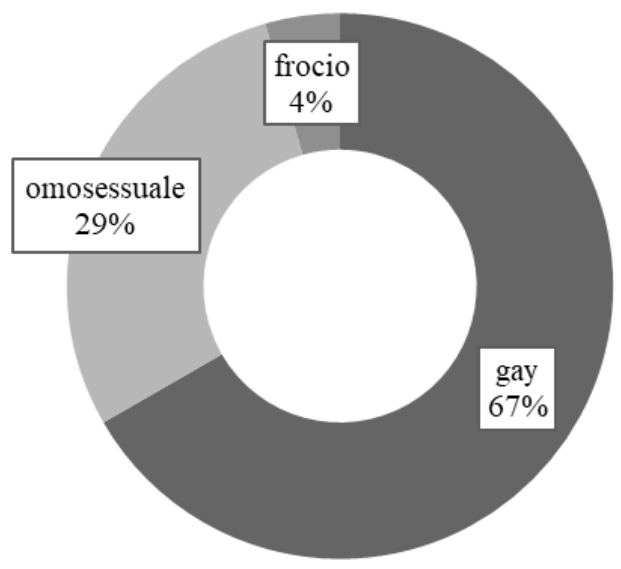

\subsection{Uso razzista della lingua italiana}

$\grave{E}$ un concetto fondato sulla presunta differenza tra le razze umane secondo la quale alcune sono biologicamente e storicamente superiori ad altre razze. Di conseguenza certe razze vengono discriminate e perseguite per garantire una 'purezza' delle 'razze superiori'. Questa teoria della distinzione tra razze superiori e inferiori è un fenomeno relativamente recente (basti pensare all'ideologia nazista del Terzo Reich). La tendenza, invece, a discriminare (nazioni, culture, classi sociali inferiori) è antichissima e proprio su questa tendenza si basa il fenomeno del razzismo per giustificare ogni tipo di discriminazione e oppressione. "Il razzismo, ovviamente, è legato a filo doppio alla xenofobia. Il primo marca le distanze in base al colore della

${ }^{14}$ La scelta di 'gay' nei titoli giornalistici potrebbe essere l'effetto dello stranierismo per suscitare l'interesse dei lettori e anche per il bisogno di essere brevi, concisi e sensazionalistici. 
pelle e ad altri tratti somatici, il secondo in base all'origine dell'altro, alla sua provenienza geografica [...]" (Arcangeli 2012: 125).

In Italia probabilmente i problemi maggiori del razzismo sono quelli che riguardano gli extracomunitari e gli immigranti in generale ${ }^{15}$. La nostra analisi del politicamente corretto nella lingua italiana si rivolgerà a quanto si è diffusa nell'uso l'espressione politicamente corretta 'di colore' per chi è 'nero' per osservare quale delle due si usa più frequentemente. L'altro argomento verterà intorno alle denominazioni per il popolo rom, vale a dire 'zingari' e/o 'nomadi'. Per questa categoria sono stati estratti 175 esempi: 67 CS e 108 da RP (si veda la Tabella 4).

\begin{tabular}{|c|c|c|}
\hline \multirow{2}{*}{} & Corriere della Serra & La Repubblica \\
\cline { 2 - 3 } & $f$ & $f$ \\
\hline di colore & 25 & 9 \\
\hline nero & 16 & 48 \\
\hline \multicolumn{2}{|c|}{} \\
\hline rom & 13 & 19 \\
\hline zingaro & 2 & 13 \\
\hline nomade & 6 & 14 \\
\hline totale & 67 & 108 \\
\hline
\end{tabular}

Tabella 4. Espressioni per l'uso razzista nei due quotidiani esaminati

Per quanto riguarda le espressioni 'di colore' e 'nero' si potrebbe osservare che si usano con alcune differenze che dipendono dal quotidiano. Dal Corriere della Sera è stato ricavato il maggior numero della espressione 'di colore' (es. 22), mentre da La Repubblica sono stati estratti più esempi della parola 'nero' (es. 23). Nella lingua italiana la differenza tra queste due espressioni, contestualmente non ha la stessa visione come nel mondo anglofono o più precisamente, statunitense dove esistono le polemiche intorno all'uso delle espressioni black e/o nigger. Per ragioni socioculturali e per il fenomeno della schiavitù negli Stati Uniti, la parola nigger ha una forte connotazione negativa e spregiativa. Non è opportuno dire neanche black, riferendosi al colore della pelle, perché ritenuto politicamente scorretto. Le

${ }^{15}$ L'Italia affronta quotidianamente il fenomeno dell'immigrazione e perciò nei quotidiani si trovano numerosi articoli su questo tema. L'argomento è troppo complesso per essere affrontato in questa sede per cui rimandiamo ad alcuni siti che contengono diversi titoli da consultare: <http://www.immigrazione.biz/libri.php $>,<$ https://www.unilibro. it/libri/f/argomento/immigrazione>, 7/2/2019). 
persone di colore dovrebbero essere nominate African-American. In Italia, la parola 'negro' non è mai stata offensiva, ma sotto l'influsso del mondo anglofono, oggi è diventata una parola 'da evitare'. Invece, 'nero' non porta nessuna connotazione negativa e perciò si usa normalmente e comunemente nella lingua italiana. Si può notare che l'espressione politicamente corretta 'di colore' (35\%) si è ormai abbastanza diffusa nella lingua italiana ed è entrata nell'uso frequente (si veda il Grafico 5).

(22) «Non volevano colpire me come Daisy, ma in quanto ragazza $d i$ colore». (CS 30/7/2018)

(23) Il sindacalista nero Aboubakar Soumahoro ha denunciato che a Roma non riesce ad affittare casa in quanto nero. (RP 25/7/2018)

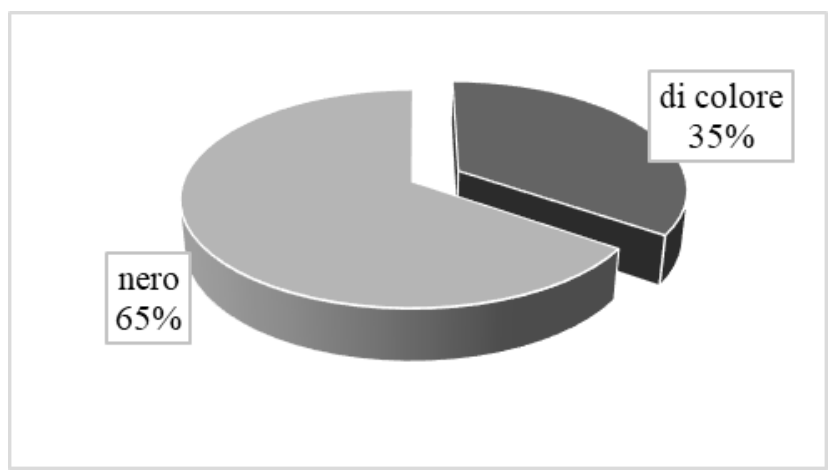

Grafico 5. Espressioni per l'uso razzista - il colore della pelle

Fra tutte le etnie o minoranze in Italia, quella dei 'rom' è forse la più "stigmatizzata". Dal corpus delle parole 'rom', 'zingaro' e 'nomade' si può osservare che tutte e tre le parole sono in uso (si veda il Grafico 6).

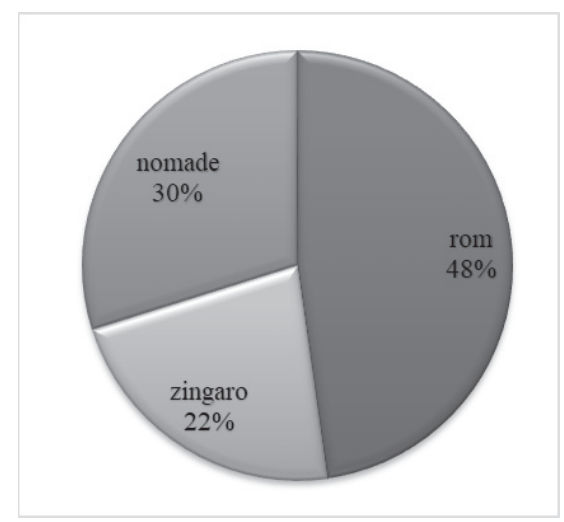

Grafico 6. Espressioni per l'uso razzista - l'appartenenza ad un popolo 
Nel Corriere della Sera, comunque, sono stati rintracciati soltanto due esempi della parola 'zingaro' e inoltre all'interno delle citazioni di persone che dicevano di essere state chiamate in quel modo e descrivevano quell'avvenimento come qualcosa di negativo e dispregiativo (es. 24). Essere chiamato 'zingaro' per le persone in questione porta una connotazione dispregiativa e lo stesso dimostrano anche gli altri esempi che contengono questa parola. La parola 'rom' (es. 25) si usa più frequentemente (48\%) negli articoli analizzati perché rappresenta la denominazione neutrale ${ }^{16}$ per questi gruppi di migranti e nomadi. Lo stesso si potrebbe dire per la parola 'nomade' (es. 26) che comprende 1/3 degli esempi; essa semplicemente "implica spostamenti periodici per la sopravvivenza e la riproduzione del gruppo"17 e viene percepita come politicamente corretta.

(24) «Invece ho ricevuto una montagna di insulti, qualcuno mi ha dato dello zingaro». (CS 5/7/2018)

(25) "Gli stranieri, i rom, i gay: sono i primi scalpi da esibire in questo ritorno della fiamma del razzismo [...]". (RP 26/7/2018)

(26) Gipsy, nomade e napoletano: l'incontro tra la tradizione rom e quella dell'Italia meridionale [...]. (CS 20/7/2018)

\section{CONCLUSIONE}

Il politicamente corretto concerne vari aspetti della vita umana concentrandosi principalmente nell'eliminare le espressioni considerate discriminatorie, spregiative e connotate negativamente, cercando in questo modo di non offendere nessuno. Dopo l'analisi sull'uso del politicamente corretto nei due quotidiani italiani si può concludere che il fenomeno non si è diffuso così tanto e che esiste un largo uso di forme sessiste nonostante le alternative proposte dai linguisti e dalla legislazione. Alcuni campi, però, sono stati sottoposti a cambiamenti.

Per quanto riguarda l'eufemizzazione, si può osservare che alcune proposte del linguaggio politicamente corretto sono state accettate, per esempio la prevalenza dell' espressione 'disabile' della quale è stato raccolto il maggior numero di occorrenze rispetto agli altri esempi. Per le disabilità visive e uditive invece non si può dire lo stesso, perché dai dati ottenuti le espressioni 'sordo' e 'cieco' comunque si usano più frequentemente negli articoli analizzati. I nomi delle professioni sono cambiati per lo più per farli coincidere con i mestieri d'oggi ('agenti della polizia penitenziaria')

${ }^{16}$ Rom - Insieme di gruppi migranti e nomadi diffusi in tutto il continente europeo e nelle Americhe. Tratto dal sito: <http:/www.treccani.it/enciclopedia/rom> (7/2/2019).

${ }^{17}$ Tratto dal sito: $<$ http://www.treccani.it/enciclopedia/ricerca/nomade/> (7/2/2019). 
e per eliminare le connotazioni negative che alcune esprimono ('operatore ecologico'). Il sessismo nella lingua italiana rappresenta un problema che è stato riconosciuto da diversi studiosi e dalla legislazione ${ }^{18}$. Comunque, il riconoscimento da solo non basta perché bisogna anche combattere gli stereotipi tradizionali sul piano sociale. Anche se la società ha già inserito nella legislazione delle regole precise rispetto all'attenzione all'uso linguistico, sono ancora piuttosto forti le resistenze del discorso mediatico nei confronti dell'uso dei femminili regolari in riferimento a donne che svolgono funzioni un tempo esclusivamente maschili. I nomi come l'avvocata, la sindaca, la ministra, la giudice sono femminili perfettamente regolari, ma ancora non si sono completamente affermati. Dai dati si rivela la prevista onnipresenza del genere maschile come genere neutro che si usa anche per riferirsi a gruppi di persone di entrambi i sessi. Per quanto riguarda l'uso omofobo della lingua, la parola 'gay', considerata più politicamente corretta, si è rivelata di uso frequente negli articoli analizzati (più di $2 / 3$ di tutte le espressioni dell'uso omofobo). Nonostante il fatto che la parola 'nero' non porti nessuna connotazione negativa e si usi comunemente nella lingua italiana $(65 \%)$, anche l'espressione politicamente corretta 'di colore' si è ormai diffusa ed è entrata nell'uso, come dimostra il $35 \%$ degli esempi estratti che comprendono le espressioni di uso razzista della lingua italiana. La parola 'rom', sentita come neutra, si usa più frequentemente $(48 \%)$ rispetto a 'nomade' $(30 \%)$ e a 'zingaro' $(22 \%)$ che è fortemente spregiativo.

Anche se, come sostengono alcuni autori, il PC si limita a intervenire sulla forma piuttosto che sulla sostanza dei problemi (cfr. Canobbio 2009), con la presente ricerca abbiamo provato che alcuni esempi di PC non sono affatto estranei alla lingua italiana ('gay', 'disabile', 'di colore', 'colf' ed altri). Il fenomeno del PC non ha profondamente segnato la lingua d'oggi, soprattutto per quanto riguarda la categoria del sessismo linguistico, ma un certo cambiamento di mentalità e di comportamenti linguistici si è comunque verificato.

${ }^{18}$ Atti legislativi che si riferiscono all'uso ufficiale della lingua, soprattutto le norme sulla lingua rivolte alla pubblica amministrazione (Codice di stile delle comunicazioni scritte ad uso delle amministrazioni pubbliche pubblicato dal Dipartimento per la Funzione Pubblica della Presidenza del Consiglio dei Ministri, 1993; Manuale di Stile. Strumenti per semplificare il linguaggio delle amministrazioni pubbliche. Proposta e materiali di studio, a cura di Alfredo Fioritto, 1997; già citata Robustelli, Linee guida per l'uso del genere nel linguaggio amministrativo, 2012; Linee Guida per l'uso del genere nel linguaggio amministrativo del MIUR, 2017 e molti altri sia nazionali che regionali). 


\section{BIBLIOGRAFIA}

Antonelli, G. (2007). L'italiano nella società della comunicazione 2.0. Bologna: il Mulino.

Aprile, M. (2005). Dalle parole ai dizionari. Bologna: il Mulino.

Arcangeli, M. (2012). Cercarsi Dante disperatamente. L'italiano alla deriva. Roma: Carocci.

Canobbio, S. (2009). Confini invisibili: l'interdizione linguistica nell'Italia contemporanea. In G. Iannàcaro e V. Matera (a cura di), La lingua come cultura (pp. 35-47). Torino: Utet.

Codice di stile delle comunicazioni scritte ad uso delle amministrazioni pubbliche (1993). Elaborato da un gruppo di giuristi e linguisti nominato da Sabino Cassese, all'epoca Ministro per la Funzione Pubblica. Pubblicato dal Dipartimento per la Funzione Pubblica della Presidenza del Consiglio dei Ministri.

Crisafulli, E. (2004). Igiene Verbale, Il politicamente corretto e la libertà linguistica. Firenze: Vallecchi.

Faloppa, F. (2011). Razzisti a parole (per tacer dei fatti). Bari: Gius. Laterza \& Figli.

Fioritto, A. (a cura di). (1997). Manuale di stile: strumenti per semplificare il linguaggio delle amministrazioni pubbliche. Presidenza del Consiglio dei Ministri, Dipartimento della Funzione Pubblica. Bologna: Il Mulino.

Fornara, O. (2009). Il linguaggio non sessista in Italia. Posizioni istituzionali e pratiche d'uso. In G. Giusti e S. Regazzoni (a cura di), Mi fai male... Atti del Convegno, Venezia, Auditorium Santa Margherita, 18-19-20 novembre 2008 (pp. 150-163). Venezia: Cafoscarina.

Galić, B. (2011). Rodna ravnopravnost i diskriminacija u Hrvatskoj: istraživanje „Percepcija, iskustva i stavovi o rodnoj diskriminaciji u Republici Hrvatskoj", Ured za ravnopravnost spolova Republike Hrvatske. Zagreb: Biblioteka Ona.

Galli de' Paratesi, N. (1964). Le brutte parole, Semantica dell'eufemismo. Torino: Mondadori.

Hughes, G. (2010). Political Correctness. A history of Semantics and Culture. Chichester: Wiley-Blackwell.

Lepschy, G. (1989). Lingua e sessismo. Nuovi saggi di linguistica italiana (pp. 61-84). Bologna: il Mulino (trad. it. di Lepschy G. (1987). Sexism and the Italian language. The Italianist, 7 (pp. 158-169), con un'appendice su Sessismo e lingua dei giornali).

Lepschy, A., Lepschy, G. e Sanson, H. (2001). Lingua italiana e femminile. Quaderns d'Italià, 6, 9-18. 
Lepschy, A., Lepschy, G. e Sanson, H. (2002). A proposito di -essa. L'Accademia della Crusca per Giovanni Nencioni (pp. 397-409). Firenze: Le Lettere.

Linee Guida per l'uso del genere nel linguaggio amministrativo del MIUR (2017). A cura di Cecilia Robustelli, coordinatrice del Gruppo di lavoro del MIUR.

Robustelli C. (2000). Lingua e identità di genere. Studi Italiani di Linguistica Teorica e Applicata, XXIX, 507-527.

Robustelli, C. (2012). Linee guida per l'uso del genere nel linguaggio amministrativo. Firenze: Regione Toscana / Accademia della Crusca.

Robustelli, C. (2018). Lingua italiana e questioni di genere. Riflessi linguistici di un mutamento socioculturale. Roma: Aracne.

Sabatini, A. (1987/1993). Il sessismo nella lingua italiana. Commissione nazionale per la realizzazione della parità tra uomo e donna. Roma: Presidenza del Consiglio dei Ministri (con la collaborazione di Marcella Mariani e la partecipazione alla ricerca di Edda Billi, Alda Santangelo).

Sensini, M. (2009). La grammatica della lingua italiana. Milano: Mondadori.

\title{
Sitografia
}

http://www.treccani.it/enciclopedia (20/5/2018)

http://www.treccani.it/vocabolario (10/6/2018)

\author{
POLITICAL CORRECTNESS IN TWO MAJOR ITALIAN \\ NEWSPAPERS
}

\section{Summary}

This study deals with the phenomenon of political correctness in contemporary Italian. The effects of political correctness in the language of Italian newspapers are observed, along with the areas it has influenced and areas that have been subject to changes and denominations in terms and expressions. In this regard, four main categories have been introduced with their definitions and origins. The research section consists in extracting examples that determine the use of political correctness in contemporary Italian. Articles from two Italian daily online newspapers, Corriere della Sera and La Repubblica, were examined over the course of July, 2018. Examples of terms and expressions of political correctness were extracted. They refer to: a) euphemisation, with two subcategories - one regarding people's disabilities and another regarding the names of certain professions; $b$ ) the use of sexist forms; c) homophobic use; and d) the racist use of Italian. These examples 
are analysed and discussed in order to observe how and to what extent certain fields have undergone linguistic change.

Keywords: political correctness, Italian newspapers, euphemisation, sexism, homophobia, racism. 